\title{
Assessment of Health Management Information System Practice on HIV/AIDS Related Commodities in Public Health Facility Found in Harar Town, Ethiopia
}

\author{
Ahmed Bedru ${ }^{1}$, Rebik Shukure ${ }^{2,}$, Kalkidan Derese ${ }^{1}$ \\ ${ }^{1}$ Department of Pharmacy, Haramaya University, Harar, Ethiopia \\ ${ }^{2}$ Department of Nursing, Salale University, Oromia, Ethiopia \\ Email address: \\ rebishukure92@gmail.com (R. Shukure) \\ ${ }^{*}$ Corresponding author
}

\section{To cite this article:}

Ahmed Bedru, Rebik Shukure, Kalkidan Derese. Assessment of Health Management Information System Practice on HIV/AIDS Related Commodities in Public Health Facility Found in Harar Town, Ethiopia. International Journal of Sustainability Management and Information Technologies. Vol. 5, No. 1, 2019, pp. 1-7. doi: 10.11648/j.ijsmit.20190501.11

Received: December 21, 2018; Accepted: January 24, 2019; Published: March 11, 2019

\begin{abstract}
Health information systems generate information to inform health planners and decision makers on what is happening at the delivery facilities. Health Management Information System (HMIS) improves health management; which is pre-requisite for good health care delivery services. HMIS is there to fill the gap between disease occurrences or health problems and the response of the health service providers. A successful Pharmaceutical Management Information System (PMIS) is able to synthesize the large volume of data generated by pharmaceutical management operations. This study was aim to assess the practice of HMIS on HIV/AIDS related commodities in public health facilities found in Harar town, Ethiopia from March 27- April 27, 2018. The study was conducted a cross sectional study design and used quantitative data collection techniques to gather the required information. Among all health facilities majority $(87.5 \%)$ of health facilities, used HMIS in their store, while $12.5 \%$ of Health facilities was not used HMIS. Out of all public health, three fourth of health facilities (75\%) had a computer to manage patient and drug related information and $12.5 \%$ health facilities had not computer and they were used manual records for managing information in their pharmacies. One third of health facilities (62.5\%) were used daily ART register for recording information on the quantity of ART drugs dispensed. The study also revealed that one hospital was used only Stock card to record information on the quantity of ARV drugs in the store and 37.5\% were used Bin card, 50\%) were used both Stock card and Bin card. Lack of training, (Adjusted odds ratio (AOR) =3.02, 95\% CI (1.8-4.6)), lack of skilled manpower $(\mathrm{AOR}=1.9,95 \% \mathrm{CI}(1.2-2.5)$ and lack of budget $(\mathrm{AOR}=3.8,95 \% \mathrm{CI}(2.1-5.7)$ were factors having statically significant associations with Health Management Information System Practice.
\end{abstract}

Keywords: Information System, Practice, Health Management

\section{Introduction}

At the beginning, health information system were oriented only to collect information on disease and health services output, but in contemporary era health information systems are considered to be part of health system; and hold great importance in the planning and decision-making of health care delivery services. Health information systems generate information to inform health planners and decision makers on what is happening at the delivery facilities. Health Management Information System (HMIS) improves health management; which is pre-requisite for good health care delivery services. HMIS is there to fill the gap between disease occurrences or health problems and the response of the health service providers [1]. A successful Pharmaceutical Management Information System (PMIS) is able to synthesize the large volume of data generated by pharmaceutical management operations. It then processes the data into information for use in planning activities, estimating demand, allocating resources, and monitoring and evaluating pharmaceutical management operations [2].

According to the 2014 estimate, Ethiopia had 793,700 
people living with HIV with 15, 700 new HIV infections and 35, 600 AIDS-related deaths. The national HIV prevalence was $1.14 \%$ in 2014 and it is declining significantly varying by age, gender and geographical location Antiretroviral (ARV) drugs and HIV tests are both relative newcomers to public health logistics systems, and they have particular characteristics that often require making adaptations to the supply chain through which they are manage. Even about $40 \%$ of people have no information on that a person can be infected by receiving contaminated blood and $12 \%$ have no information on that direct or indirect contact are the most important routes for transmission of hospital-acquired infections. The special nature of ARV drugs and HIV tests influences the design of the inventory control and logistics management information systems, the design of the storage and distribution networks, and the process for implementing upstream and downstream functions. Because the programs that use these commodities-for example, prevention of mother-to-child transmission (PMTCT), and antiretroviral therapy (ART) - are still evolving in the way services are provided [3-5]

Drug management cycle comprises four basic components: selection, procurement, distribution and use. At the center of the pharmaceutical management cycle is the core of related management support systems including the planning and organization of services, financing and financial management, information management and human resource management. These management support systems hold the management cycle together. The entire cycle rests on a policy and legal framework that establishes and supports the public commitment to supply essential medicines [6].

The Ethiopian National Health Information System assessment report of 2007 identified that the health management information is among the major problems of the sector [7]. health information and coverage of antenatal care, postnatal care and pediatric nutrition is low in the country [8, 9]. Pharmaceutical management system at health facilities in Ethiopia is reportedly so poor that the system in place does not allow for the effective and efficient monitoring that is required to manage antiretroviral (ARV) drugs. The processes of selection, quantification, procurement and ordering, distribution, and use were not uniform throughout the country's health facilities [10]

Effective drug supply management and inventory control avoid stock out loss due to unnecessary expiry, theft and ensure that the desired pharmaceutical products are available at all times in adequate quantity. However, in many low and middle-income countries, the capacity of the HMIS has always been challenging and weak. The ARV supply chain management has become increasingly difficult due to increasing number of people on ART, increasing number of sites providing ART and a greater diversity of different ARV regimen [11].

Information technology has advantages for managing patient information, especially for ART patients and children to manage their treatment and feeding practice. However, there are some barriers such as lack of computer skills of the dispensers, lack of motivation to use technologies; they feel like as an additional work and lack of access to use information technology. Generally, there is technical, financial and professional challenges facing pharmacy practice to introduce IT [3, 12]. Currently, it is assume that the magnitude of the use of this technology by pharmacists and druggists is not fully practice in the health facilities in Ethiopia, particularly in Harar. The study was try to assess the gaps of HMIS practice on HIV/AIDS related commodities, particularly in the medication dispensing and inventory control process, and to assess the advantage of using PMIS and factors that affect to use HMIS in inventory control system

\section{Methodology}

\subsection{Study Area and Study Period}

The study was conducted in four public hospitals and four health centers, which are found in Harar town, Harari regional state, Eastern Ethiopia which is located $526 \mathrm{~km}$ from the capital city, Addis Ababa.

Harar is the capital city of Harari regional government and it is one of the oldest cities of Ethiopia. It is located in Eastern Ethiopia, $526 \mathrm{~km}$ from Addis Ababa. The State has no administrative zones. The total numbers of kebeles of the city are 19 , while the rural part of the state has 17 farmers associations. The State's size is estimated at $343.2 \mathrm{~km}^{2}$ with a total population of 183,415 among which $50 \%$ are male. It is situated at 923 latitude and 4224 longitude wide with 6100 above sea level, $90 \%$ of the region estimated to be woyenadega (between 1000 and $1500 \mathrm{~m}$ ) and rest $10 \%$ is desert (1100m) [13].

In Harari region, there are 6 hospitals from which 3 of them are under HRHB, 1 is under FMOH, and the rest 2 are private hospitals. Additionally, there are 8 health centers among which 4 of them are found in Harar town and the rest in the rural part of the city. The study was conducted from March 27 to April 27, 2018.

\subsection{Study Design}

The study was conducted a cross sectional study design and used both quantitative and qualitative data collection techniques to gather the required information.

\subsection{Source and Study Population}

All public health facilities providing, PMTCT and ART in Harar town was the source facilities. All health care professionals and all documents that were used to manage the supply chain of ARV drugs in Harar was also sources of information. All staffs working in the ART clinics, pharmacy of the four selected public hospitals and four health centers was the study population.

\subsection{Inclusion Criteria}

Public health facilities who was provided ART services. 


\subsection{Sampling Technique and Sample Size Calculations}

\subsubsection{Health Facility Selection}

The sample of health facilities was calculate by using the Logistic Indicators Assessment Tool (LIAT) for ARV drugs and Test kits of USAID/DELIVER. This document suggested that it would be enough to take $15 \%$ of the targeted health facilities as sample for the study (USAID/DELIVER PROJECT, task 1, 2009). Since Harar is a small town with limited number of public health facility, all public health facility which is found in Harar town was included in the study.

\subsubsection{Selection of Health Professionals}

Heads of pharmacies, ART dispensers, and ART coordinator from the selected hospitals and health centers was the key respondents for the study.

\subsection{Data Collection and Management}

\subsubsection{Data Collection Instruments}

The LIAT for ARV drugs and test kits was used as a data collection tool (USAID/DELIVER PROJECT, 2009). A combination of semi-structured questionnaire was used to collect data on the supply management of HIV/AIDS related commodities.

\subsubsection{Data Entry and Analysis}

The collected data was manually checked for completeness and consistencies before being entered into the computer. The quantitative data was entered and analyzed by using SPSS version 23 .

\subsection{Operational Definitions}

HIV/AIDS related commodities: refers to ARV drugs and HIV rapid Test kits.

All these components adapted from the PRISM assessment tool have a likert scale measure, ranging from "strongly disagree" to "strongly agree". Finally, health workers' mean scores were use to split health professionals'.

Health information utilization into "has good routine health information practice" or "has poor routine health information utilization practice" practices.

\subsection{Ethical Consideration}

A formal letter was obtained from school of pharmacy, college of health and medical science and had given to each health center and public hospital in order to get permission to conduct the Verbal consent from all respondents will obtained before enrolling them as the respondents of the study. During the consent process, the respondents were provide with information regarding the purpose of the study, why and how they selected as the respondents of the study, and what will expected of them. They were also informed that as they could withdraw from the study at any time during the interview process. Participants were also assure about confidentiality of the information that were obtain in the course of the study. To assure the anonymity of the respondents' personal identifiers were not use during the data collection.

\section{Result}

A total of Eight public health facilities were visited during this assessment; of which four were hospitals and four were HCs located in Harar town.

\subsection{Socio Demographic Characteristics of the Study Subjects}

There was no fair gender balance among the respondents with three males and five females. With respect to their academy or professional category one was pharmacist, three were druggist and four were nurses as shown in figure 1 below.

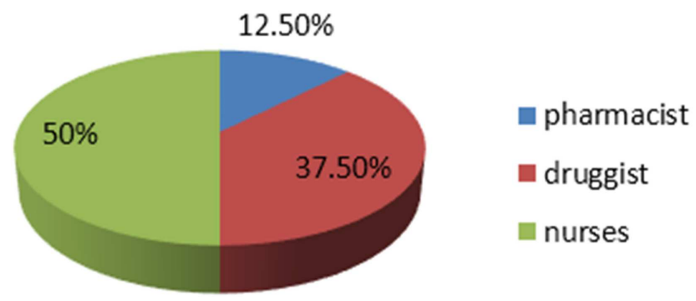

Figure 1. Professional category of respondents who work in ART clinics, pharmacy at public hospitals and health centers of Harar town.

The respondents had an age range between 23 to 42 years, with an average of 31 years. Concerning the type of institutions $50 \%$ of respondents were working at hospitals and the rest $50 \%$ are working at health centers. Respondents with 1-4 years formed the largest proportion followed by those who had served 5-8 years as shown in figure 2 below.

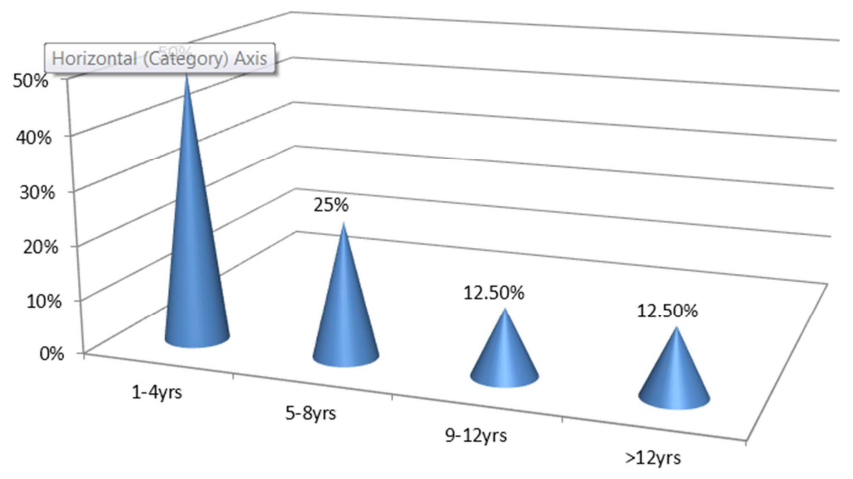

Figure 2. Years of Experience at working station.

\subsection{Questions Related To ART Service, ART Pharmacy, Storage Conditions, Stock Keeping Practice and Physical Inventory}

All of the selected facilities had a copy of STG (2014) and document that lists all the recommended ARV drug regimens to be prescribe and dispensed.

In the selected facilities the principal person responsible for managing ARV drugs were pharmacists (37.5\%), druggists $(37.5 \%)$, both pharmacists and druggists $(12.5 \%)$ 
and other health professionals (12.5\%).

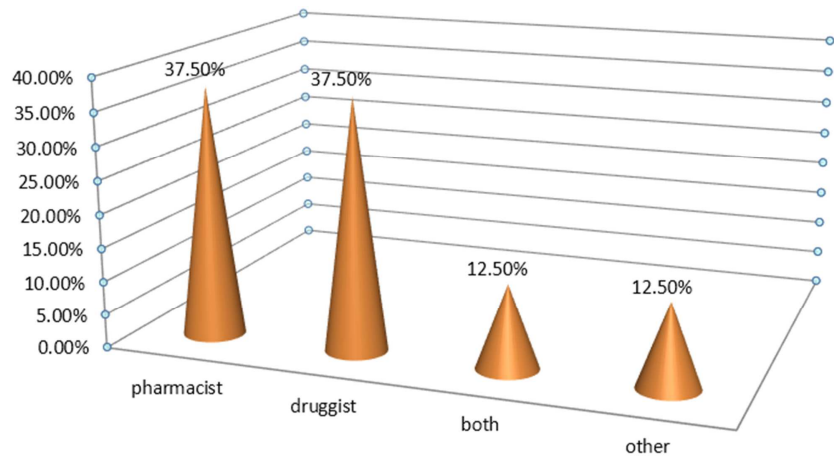

Figure 3. A principal person responsible for managing ARV drugs in public hospitals and health centers found in Harar town.

Regarding training, all health facilities provide training for personnels that give service on ART pharmacy, except a single hospital.

Majority of health facilities $62.5 \%$ were use daily ART register for recording information on the quantity of ART drugs dispensed. The study also revealed that one hospital was used only Stock card to record information on the quantity of ARV drugs in the store and two hospitals and one $\mathrm{HC}(37.5 \%)$ were used Bin card, one hospital and three $\mathrm{HC}$ $(50 \%)$ were used both Stock card and Bin card. In majority of the cases, two $(50 \%)$ of $\mathrm{HCs}$ and three $(75 \%)$ of hospitals used daily ART register to record patient by regimen information and one (25\%) of HCs used EDT to record patient by regimen information.

All the selected health facilities were providing PMTCT and PEP services in addition to ART services. all the selected HCs four $(100 \%)$ and two $(50 \%)$ of hospitals used TDF/3TC/EFV for PEP of high risk patients, while $50 \%$ of health facilities were not use any drug for PEP for low risk patients. Based on the new guideline, all of the facilities had initiated HAART for pregnant mothers irrespective of their CD4 count. Mothers were treated using TDF/3TC/EFV while NVP syrup was used to treat the infant.

In majority of cases, four (100\%) of HCs and three (75\%) of hospitals used HMIS in theire store, while a single hospital was not used HMIS due to lack of personnels that are trained by HMIS. Regarding the advantage of HMIS $50 \%$ of the respondents said that it can facilitate our daily activities and decrease work load, $25 \%$ of respondents said that it can be increase effectiveness of our job and the rest $25 \%$ of respondents said that it can be used for reporting files.

\subsection{Access for Information Technology}

At seven Health Facilities ART pharmacy, there is a Desktop computer to manage patient and drug related information $(87.5 \%)$. Out of eight HFs only one HF has internet access in the pharmacy, the rest had not internet access at their pharmacy. Sources of computers and IT equipment are mostly from their institution i.e. four HFs
(57.14\%) as shown in table1 below.

Table 1. Access for Information Technology among respondents in Government Hospital and Health Centers Pharmacies in Harar town.

\begin{tabular}{lll}
\hline Charcteristics & Frequency & percent \\
\hline Presence of computer at HFs yes & 7 & $87.5 \%$ \\
No & 1 & $12.5 \%$ \\
Type of computer desktop & 7 & $100 \%$ \\
Source of computer and IT equipment & & \\
From the institution & 4 & $57.14 \%$ \\
From NGOs & 1 & $14.28 \%$ \\
From both sources & 1 & $14.28 \%$ \\
From other source & 1 & $14.28 \%$ \\
Internet access yes & 1 & $12.5 \%$ \\
No & 7 & $87.5 \%$ \\
\hline
\end{tabular}

\subsection{Information Access Behavior in Pharmacy Practice}

When respondents were asked about the way of providing services to patients four $(50 \%)$ of the respondents used paper based recording and four (50\%) are using both paper and computer to manage patient and drug related information. Respondents who heard information about PMIS/HMIS were seven, while the rest one had not. All eight respondents perceived that IT will improve efficiency and effectiveness. Regarding the use of information recorded three (37.5\%) stated for decision making, budgeting and planning purpose, one $(12.5 \%)$ to provide feedback for supervisors or donors, two $(25 \%)$ of them stated for ease of access of patient information and the rest two (25\%) for all purpose. At all HFs there is a focal person for HMIS. At five HFs pharmacist or druggists are handling pharmacy information system and at three HFs other health professionals are responsible for handling pharmacy information management system.

Out of eight respondents seven have been trained in HMIS/PMIS while the rest one had not been trained. Among these personells who got training six $(87.5 \%)$ were both pharmacists and druggists and the other one (12.5\%) was other health professional. Dispensers are using different information sources to manage dispensing practice. From eight respondents one was using computer only while four respondents were using Patient information sheet. Response regarding appropriate and convenient tool to manage information, computer based recording is more convenient five $(62.5 \%)$ and three $(37.5 \%)$ of respondents stated both computer and manual recording are convenient.

Almost all public Health facilities in Harar town produce pharmacy reports and shared for Ministry of health and regional health bureau $12.5 \%$, for Regional Health bureau $25 \%$, for Regional Health bureau and other concerned parties (supporter NGOs) $62.5 \%$ i.e. six HFs monthly, one HF on bimonthly, one HF weekly basis produce reports. About four $(50 \%)$ respondents stated that there was a gap between the services they provide and the report generated and four $(50 \%)$ of them stated no gap. Regarding the possible reasons for this gap one respondent stated "information not fully recorded", three respondents stated "data are not properly compiled" as shown in Table 2 below. 
Table 2. Information access behavior in pharmacy practice.

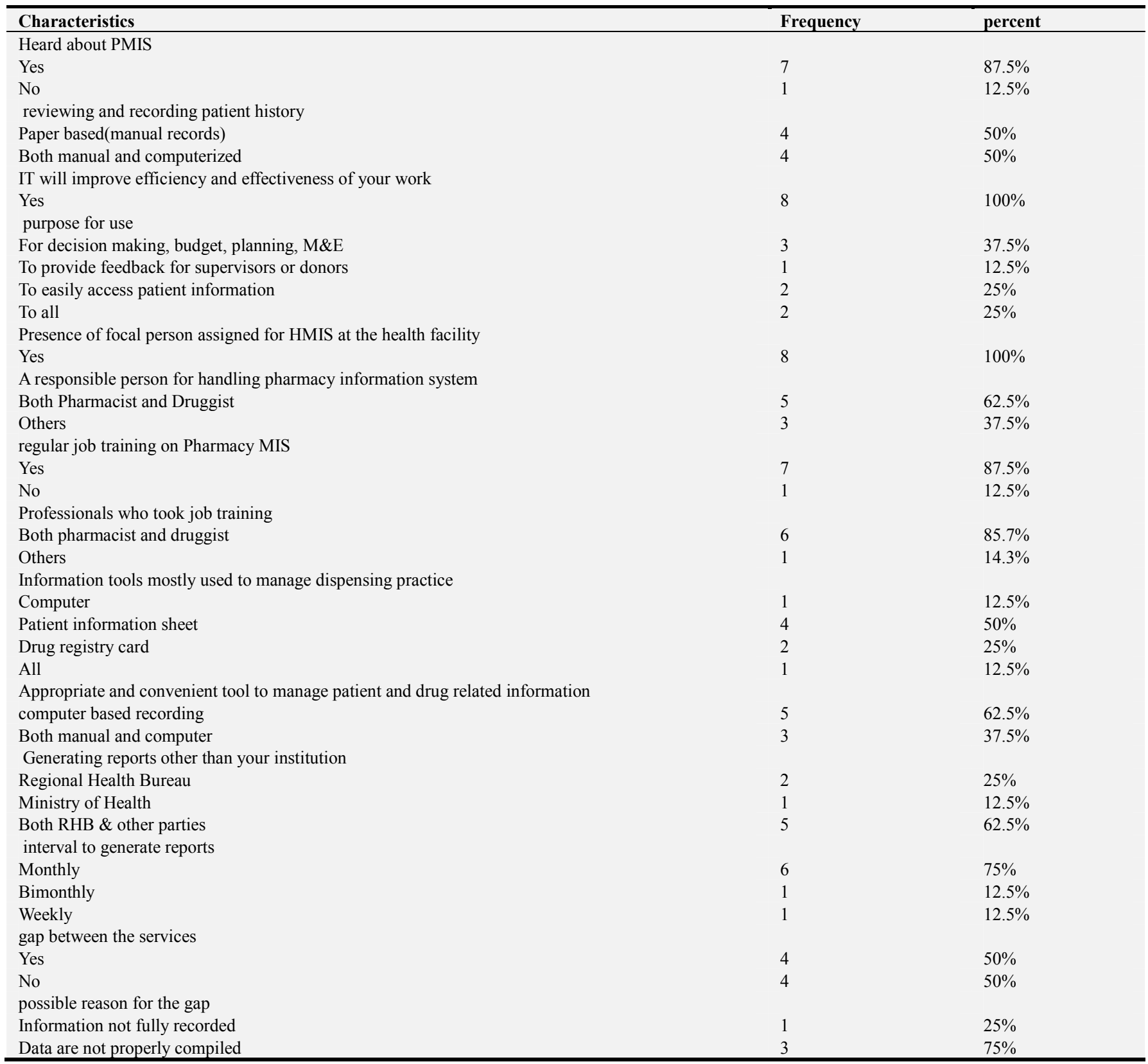

\subsection{Factors Affecting Use of Information Technology}

As indicated in Table 4.3, ten selected variables that were expect to be the possible factors that affect the utilization of information technology for pharmacy service by pharmacists and druggists. The responses were graded from "strongly agree to strongly disagree". One of the factors affecting the utilization of information technology is lack or inadequacy of know-how/training as indicated by the responses three $(37.5 \%)$ strongly agree or agree, two $(25 \%)$ respondents not sure and three (37.5\%) strongly disagreeing or disagree. Most of respondents stated that one of the factors that affect the utilization of information technology is shortage of budget, as indicated by the responses five $(62.5 \%)$ agreed or strongly agreed, three (37.5\%) not sure with the shortage of budget as a problem. The majority of respondents, five $(62.5 \%)$ agreed or strongly agreed with the questions 'Lack of skilled pharmacy personal to use information system' as a problem and two $(25 \%)$ of them disagreed on this point and one $(12.5 \%)$ not sure. Lack of interest to use information technology was another question that was asked. From eight respondents five $(62.5 \%)$ of respondents disagree and three (37.5\%) agree or strongly agree. Respondents who did not know about lack of policy or were not sure that lack of policy affect the use of IT are two (25\%), three (37.5\%) agreed or strongly agreed and three $(37.5 \%)$ disagreed or strongly disagreed that there is a lack of policy to use IT. Regarding technology transfer problem five $(62.5 \%)$ agreed or strongly agreed that there is technology transfer problem, because most professionals or administrators do not change their 
work habit or working trend, and they need to continue as they were doing. From eight respondents two (25\%) of the respondents were not sure whether there is a problem or not and one $(12.5 \%)$ of them are perceived that there is no problem for technology transfer. Five (62.5\%) of the respondents believed that there is lack of management commitment and three (37.5\%) said the management is committed to IT usage at the pharmacy. About three (37.5\%) of the professionals were not aware of the competitive advantage of IT in Pharmacy, three (37.5\%) of the respondents disagreed, because they believe that most of professional pharmacy staffs are aware of the advantages of IT and two $(25 \%)$ of the respondents were not sure. The effect of culture on the use of IT was another question and five $(62.5 \%)$ responded that there is no culture of utilization of IT, Most of the time information recording at HFs was based on manual records, two (25\%) respondents were not sure and one $(12.5 \%)$ were disagreed. Other important issue that was given attention in this study was about respondents' computer phobia or fear of using IT. With respect to this, one $(12.5 \%)$ agreed that there is a fear of using IT and most of the respondents i.e. six $(75 \%)$ disagreed with the perception of fear of using IT. This means that they were not phobic or apprehensive to use computer. However, one (12.5\%) were reserved in their attitudes.

Table 3. Multinomial logistic regression on factors affecting the use of IT at ART Pharmacies in Harar town.

\begin{tabular}{llll}
\hline Variables & Adjusted odds ratio(AOR) & $\mathbf{9 5 \%}$ confidence interval & p-value \\
\hline Lack of training & 3.02 & $1.8-4.6$ & 0.002 \\
lack of skilled manpower & 1.9 & $1.2-2.5$ & 0.04 \\
lack of budget & 3.8 & $2.1-5.7$ & 0.003 \\
\hline
\end{tabular}

\section{Discussion}

This study assessed the use of information technology by pharmacists, druggists and other health professional at Harar government health facilities. It is mainly concerned with the self-reported assessment of the professionals.

This study showed that majority of health facilities five (62.5\%) used daily ART register for recording informations on the quantity of ART drugs dispensed. Compared with a study that done in Addis Ababa shows that majority of health facilities used computer for recording informations on the quantity of ART drugs dispensed [2] This shows use of computer for recording informations on the quantity of ART drugs dispensed in public health facilities of Harar town were very poor. this might be due to lack of the habit of using computer in their daily activities and the absence of computers in some health facilities.

Lack of skilled man power is significantly associated with use of HMIS. Out of eight public health facilities, only one HF used EDT to record patient by regimen information. In contrast to a study that done in Addis Ababa shows that most (95\%) of public hospitals and health centers use EDT to manage information at their dispensary. contrast to this study, study shows that there is a very low usage of EDT in public hospitals and health centers of Harar town for managing patient information at their pharmacies. This might be due to shortage of professionals in the facility and there is phase out of institutions who was controlled the EDT at health facilities.

All the selected four $(100 \%)$ of HCs and two $(50 \%)$ of hospitals used TDF/3TC/EFV for PEP of high risk patients, while $50 \%$ of health facilities were not use any drug for PEP for low risk patients. When comparing this to other study that was done in Addis Ababa all the selected health facilities were used AZT/3TC/NVP and AZT/3TC regimen for PEP in high and low risk patients respectively. This might due to shortage of medications and high patient burden who wants this service, so they can give PEP services for only patients who have high risk.
During drug dispensing lack of training also significantly associated with use of HMIS, about $50 \%$ of the professionals use paper based information recording, but $50 \%$ are using both manual and computer for information recording. Compared to the study done in Addis Ababa, which was $65.79 \%$ of the professionals used electronic recordings [2]. This might be due to unavailability of computers in some health facilities and absence of habit to use electronic recording system.

\section{Conclusion}

The majority of respondents perceived the advantages of using IT at pharmacy. The study specified less utilization status of IT for pharmacy practice services in Harar, Ethiopia. The findings also indicates the need for computer training related to pharmacy profession in health care system, particularly in pharmacy practice. Lack of training, lack of skilled manpower and lack of budget were significantly associated with use of HIMS for pharmacy practice.

\section{References}

[1] WHO Global HIV/AIDS Response - Epidemic update and health sector progress towards Universal Access - 2011 Progress Report. Geneva, 2012.

[2] Mekdes A. Implementation of Anti-Retroviral Therapy (ART) Pharmacy Management Information System in Public Health Facilities in Ethiopia, 2013.

[3] USAID | DELIVER PROJECT, Task Order 1 Logistics Indicators Assessment Tool (LIAT): Antiretroviral Drugs. Arlington, Va.: USAID | DELIVER PROJECT, Task Order 2009.

[4] Suoud J, Rebik S (2018) Assessment of Knowledge and Practice towards Blood Donation among Gedebano Secondary and Preparatory School Students, Mehal Amba, SNNPR, Ethiopia. J Blood Disord Med 3 (1): dx.doi.org/10.16966/2471-5026.120. 
[5] Suoud Jemal. Knowledge and Practice of Hand Washing among Health Professionals in Dubti Referral Hospital, Dubti, Afar, North East Ethiopia. Advances in Preventive Medicine; 2018.

[6] MSH. Managing Drug Supply Training Series, 2nd ed, Participant's Guide, 2009. Available at: http://erc.msh.org/newpages/english/drugs/intro_pg.pdf. Accessed on: 22/12/2017.

[7] Ethiopian Federal Ministry of Health; guideline for implantation of the antiretroviral therapy program in Ethiopia, 2007.

[8] Rebik Shukure, Tesfahun Simegnew. Knowledge Towards Antenatal Care and Service Utilization Among Women in Fiche Town, North Shewa, Ethiopia. Advances in Materials. Vol. 4, No. 1, 2018, pp. 1-5. doi: 10.11648/j.ajp.20180401.11

[9] Rebik Shukure, Hussen Mohammed, Abdulselam Wudmetas, Sara Mohammed. Assessment of Knowledge and Factors Affecting Utilization of Postnatal Care in Fiche Town, Oromia Region, Ethiopia. International Journal of Clinical Dermatology. Vol. 1, No. 2, 2018, pp. 28-33. doi: 10.11648/j.ijcd.20180102.11.
[10] Alemayehu L. Assessment of Supply Management Current Status for Antiretroviral Therapy (ART) in Oromia National Regional State, Ethiopia. Ethiopian Pharmaceutical Association, Book of Abstract. 2009.: http://epaethiopia.org/images/k2/38/32/abstract pdf. Accessed on: $09 / 02 / 2018$.

[11] Erik JS, Andreas J, Anne BS, Simon DM, Anthony DH, Francis A Antiretroviral drug supply challenges in the era of scaling up ART in Malawi, 2011. JInt AIDS Soc;14Suppl $1:$ S4.

[12] Rebik Shukure. Assessment of Knowledge, Attitude and Practice on Timely Initiation of Complementary Feeding Among Under Two YearsChildren in Fiche Town, North Showa Zone, Ethiopia. International Journal of Biomedical Engineering and Clinical Science.Vol. 3, No. 6, 2017, pp. 103109. doi: 10.11648/j.ijbecs.20170306.16.

[13] Demographic and Health Survey. Addis Ababa, Ethiopia (2011). 